\title{
Research on the access and security Technology of Radio-on-Fiber in Power Communication
}

\author{
Sheng $\mathrm{Xu}^{[1]}$, Yijie $\mathrm{Wu}^{[1]}$, Jia Tao ${ }^{[1]}$ \\ Nanjing IOT and IC design Innovation Center Ltd.
}

\begin{abstract}
With the optical fiber communication and wireless communication technology advantage, Radio-on-Fiber (RoF) technology is a good way to solve the problems of bandwidth flexibility security and the electromagnetic interference, and its related technologies have attracted wide attention. With the construction of the Smart Grid, the bandwidth, stability and access reliability were demanded increasingly. According to the power system communication status and characteristics, transplant the RoF technology reasonably; combine with the advantages of fiber and wireless, will get the broadband and flexible information access, while reducing costs. The structure and application of hybrid RoF broadband access technology in the power system were discussed, and the corresponding performance and function were tested, which prove the power RoF technology has wide application.
\end{abstract}

Keywords-Power communication; Radio-on-Fiber; Access; Security; EPON

\section{INTRODUCTION}

At present, EPON technology was widely used in intelligent communication of power distribution systems [1], but it requires laying much fiber to connect a large number of end users directly, and the infrastructure construction cost was so high[2][3]. In the wireless access, The Wi-Fi technology (IEEE802.11 series of international standards) and WiMAX (IEEE802.16d/e series of international standards) were developing rapidly. Wi-Fi and WiMAX technologies, there are two modes, one is a basic implementation of the "multipoint" group network communication mode, and the other is based on the multihop Mesh mode [4]. Wireless access network is relatively low average cost of a single user, and can provide end users with flexible, ubiquitous access. However, the wireless access systems need many access points or base stations, that typically has to build a broadband fiber-optic network to connect to the core network [5] [6], which is still not an effective way to save the initial investment.

Taking into account the fiber-optic network and wireless communications together, use the mature technology of EPON to get the wireless service nodes together, so it could upgrade on the basis of the EPON systems, the fiber repeated investment can be saved, and because the use of fiber-optic trunk network professional communication, the security also has better protection.

\section{TECHNICAL PRINCIPLE}

RoF communication technology also has a broad application prospects in electric power communication system, the application of RoF in the power distribution systems could solve the problems of the fiber cable laying and excavation, and the problems of transmission delay and insufficient bandwidth [7].will also be able to overcome. As a special fiber and wireless network communication, it could be a good method to meet the information and communication security demand in electric power systems, which could be used as a reliable and effective solution to replace the public wireless communication

Be different with the traditional wireless communication networks, RoF communication system do not need the continuous wide networks covering large areas, but according to actual needs, build a small local area network consisting of single base station or a small amount of base stations [1] [8].The use of these small local area networks, can form a continuous or discontinuous coverage, and provide users with different services [9].

In the optical access technology used in power system, undoubtedly, the current dominant technology is the passive optical network (PON) technology [3].Based on the current technical standards, from the OLT to ONU the maximum $20 \mathrm{~km}$ transmission distance can be achieved. For example, the time-division-multiplex PON (TDM-PON), which is the most widely commercial used, can support 64 users, 1-10 $\mathrm{GB} / \mathrm{s}$ bandwidth. The wireless communications, the existing mainly technologies are: the Wireless Fidelity (WiFi), the Worldwide Interoperability for Microwave Access (WiMAX) and cellular networks (2G/3G). At present, the most popular wireless communication technology comes as WiFi technology based on 1EEE802.11a/b/g, and based on the IEEE802.16 standard, WiMAX extends the maximum working distance to become the most potential of wireless communication technology. $3 \mathrm{G}$ technology was popularized gradually, providing a good communication platform for data, voice and video multimedia services.

RoF is an advantage combination of fiber and wireless, the backbone network from the OLT to ONU, using the PON network. In the level from ONU to the target users, "gateway routing" technology is utilized, which was the most commonly used [2], Network structure shown in Figure 1. 


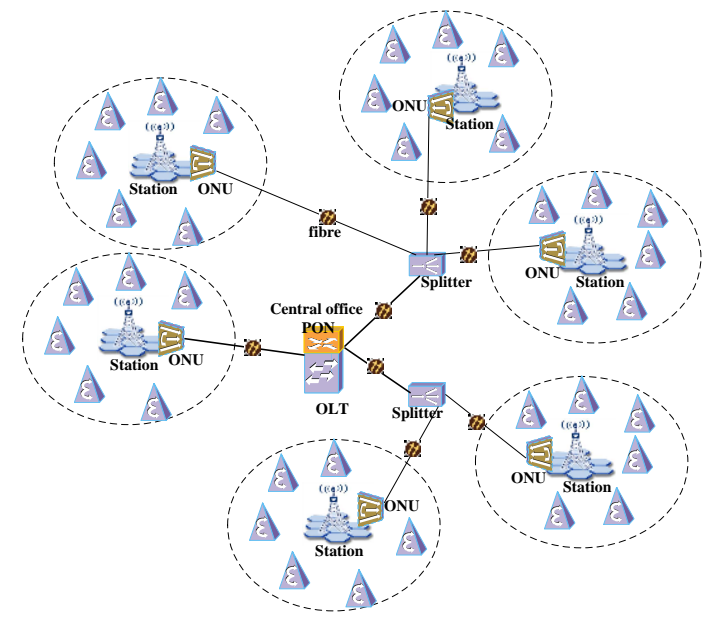

Figure 1. Network structure

It could be seen from Figure 1, a RoF access system contains the front end of wireless access systems and the back-end optical network [10]. Standard PON optical network structure was used, the current is TDM-PON, and in the future will be the WDM-PON or WDM/TDM-PON. The PON system is consisted of OLT, splitter (optical power splitter or wavelength splitters), ONU and the fiber interconnection. In this system, ONU will be directly connected to the wireless base station (BS). Base station, connected ONU directly is used as a wireless gateway, because it is the gateway between the wireless and optical parts. In addition to the gateway, the front end of wireless part uses the rest of the wireless router/base station to manage the network effectively. The front-end part of RoF access system is essentially a multi-hop wireless Grid (Mesh) network, which contains several wireless routers and a few gateways (connected to the ONU, and then connect to the Internet through OLT). Wireless part chooses the standardized technology. As the ONU is far from the central office, it can be expected that through a wireless base station (BS) re-use the spectrum in a small range effectively, very high bandwidth could obtained. Thus, RoF has the ability to meet the high bandwidth demand of a large number of wireless users.

The advantages of RoF access network are:

(1) Inherits the advantages of optical networks: the system use the high-capacity fiber-optic network as backbone, so it will have much larger capacity than the wireless networks;

(2) Inherits the advantages of wireless networks: the wirelesses parts interconnect seamlessly, so the RoF network has more flexibility than the fiber network, achieve any time, anywhere access for users;

(3) Cost advantage: In comparison with the fiber network, RoF has great cost advantages. If FTTH need to be achieved, high installation and maintenance costs are usually unbearable for ordinary users. The RoF structure does not require expensive FTTH connections [1][9], so use the hybrid access method could get the technology advantages of optical communication, but also get the cost advantage of wireless communications;
(4) "Self-organizing" advantage: as shown in Figure 1, if choose the fiber network model, once the fiber line from a splitter to a ONU was broken, the ONU and OLT user will be unable to communicate. Worst case is the line from the OLT to a splitter was broken; and all of the ONU OLT would not work. However, in RoF system, once the link breaks, the communication terminals have the ability to selforganizing the multi-hop mesh topology, using the wireless connection contact with other adjacent ONU self-adaptively, to maintain the communication. While the terminal can connect with its adjacent ONU, if an ONU interrupt or block, the system will regulate the network load automatically.

\section{TECHNOLOGY ACHIEVEMENT}

RoF in electricity power system can solve the cable laying and excavation problems, but also overcome the delay of wireless multi-hop transmission base station and bandwidth shortage. As a special network communication, RoF is a good solution to security challenges, can be an alternative solution to the public wireless network communications, the achievement in the distribution system architecture is shown in Figure 2.

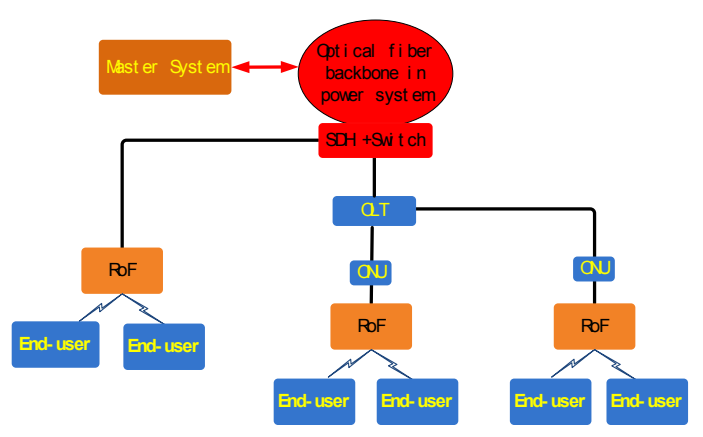

Figure 2. RoF Access structure in power system

From the Figure, RoF system, there are two access methods [8], one is the wireless base stations directly connected to the core switch, provide the air interface for the end-user services; the other is connect the RoF base station in the ONU devices of XPON Networks, also provide the air interface for the end-user services. Specific connection can be the need for flexibility.

In typical power RoF access network architecture, the wireless terminal equipment sends packet data to a neighbor's wireless router [8], and then the router will set the packet data into the Wireless Mesh Networks, the packet data will probably jump through a gateway/ONU and ultimately through the optical part of RoF system to the OLT (central office).

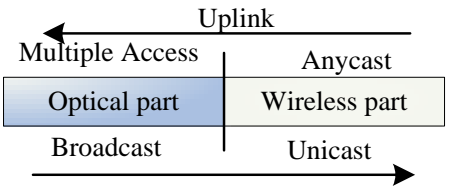

Figure 3. RoF transfer protocol base on $\mathrm{xPON}$ 
In the RoF system based on the XPON, the upstream direction (from the wireless user terminal to a gateway/ONU) in the wireless part, is an arbitrary broadcast network, that a end user can provide group to any gateway, in the optical part, the upstream direction (from ONU to OLT) is a multiaccess network, using the multi-point control protocol to avoid the conflict, and shared uplink resources. The downstream direction (from the gateway/ONU to a wireless terminal) in the wireless part is a single broadcast network, that a packet data, sent by the gateway, reaches only one destination (or user) [6]. But in the optical part, the downstream direction (from OLT to ONU) is a broadcast network, the packet sent to a particular ONU, is broadcast to all ONUs, only the particular ONU select to process. Figure 3 describes this set of RoF transmission protocol.

RoF broadband access can provide wireless coverage mode [1] [10] contains:

a) One base station coverage: coverage for independent region, such as individual or separate residential area.

b) Multi-base station but not continuous coverage: coverage for the existence of multiple independent areas, such as the multi-residential buildings in residential or more separate cell.

c) Multi-station continuous coverage: coverage for a continuous area, and provide value-added services on the conditions of continuous coverage.

d) Mixed coverage: comprehensive coverage of these three kinds of ways, according to the need for flexible coverage.

\section{SECURITY PRotection}

Major security risk RoF system may face:

a) Access to wireless may be illegal;

b) The end-user identity is fraudulent;

c) Unauthorized access into the network;

d) Data transmission is theft.

The RoF transmission backbone is the fiber optic private network, with a high degree of safety, so the security protection should be for the special application type, the main considerations is the access channel border and the RoF strengthen security. Main methods are:

a) Place the security access platform at the border, authenticate the access terminal and establish an encrypted channel to ensure security of data transmission;

b) Use the PKM technology to authenticate the access terminal;

c) Establish an encrypted channel to ensure security of data transmission.

\section{FIELD APPLICATION TEST}

To verify the practicality of power RoF, the performance test was taken at the $110 \mathrm{kV}$ "KaiFa" Transformer substation of the power company in Yangzhou. The specific tests include: the base station coverage in different terrain conditions, signal strength, terminal access peak rate, average rate, access stability and other related technical parameters.

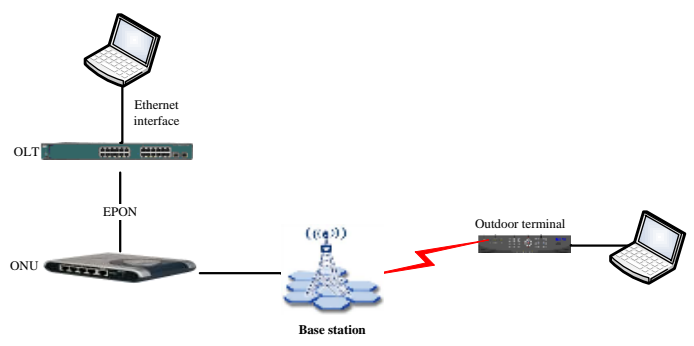

Figure 4. Performance testing structure

Using the laptop to connect the outdoor terminal, run the wireless test software, test and record the wireless parameters of access terminal in different distances from the base station, include the Receive Signal Strength Indication (RSSI), the terminal access to the peak rate, the average rate, etc., and locate the latitude and longitude to calculate the distance from the base stations. The wireless base station power is $1 \mathrm{~W}$, and forms the following record form:

TABLE I. ROF PERFORMANCE TEST RESULTS

\begin{tabular}{|l|l|l|l|l|l|}
\hline No. & Dist. & RSSI & SNR & $\begin{array}{c}\text { Max } \\
\text { uplink } \\
\text { rate }\end{array}$ & $\begin{array}{c}\text { Max } \\
\text { downlink } \\
\text { rate }\end{array}$ \\
\hline 1 & $300 \mathrm{~m}$ & $-55 \mathrm{DB}$ & $29 \mathrm{DB}$ & $2.2 \mathrm{Mbps}$ & $5 \mathrm{Mbps}$ \\
\hline 2 & $500 \mathrm{~m}$ & $-67 \mathrm{DB}$ & $23 \mathrm{DB}$ & $2.3 \mathrm{Mbps}$ & $5.09 \mathrm{Mbps}$ \\
\hline 3 & $900 \mathrm{~m}$ & $-89 \mathrm{DB}$ & $20 \mathrm{DB}$ & $1.85 \mathrm{Mbps}$ & $4.18 \mathrm{Mbps}$ \\
\hline 4 & $1200 \mathrm{~m}$ & $-90 \mathrm{DB}$ & $19 \mathrm{DB}$ & $1.71 \mathrm{Mbps}$ & $4.01 \mathrm{Mbps}$ \\
\hline
\end{tabular}

To verify the functions of the power RoF broadband access, the voice and video transmission also be tested. The test system architecture is shown in Figure 5.

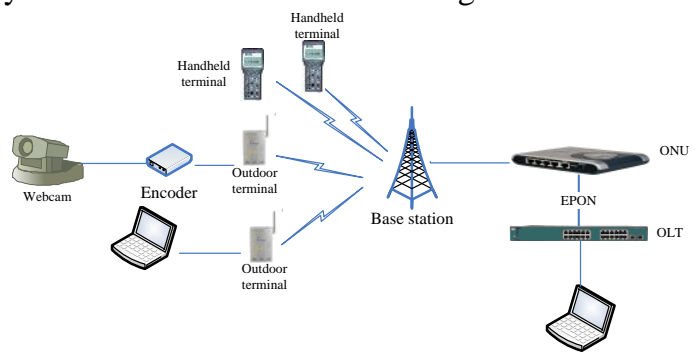

Figure 5. Speech video function test

Two testers, carrying hand-held terminals, moved eastward and westward separately, tested the wireless link coverage, voice communications, the distance measured by latitude and longitude. A laptop computer accesses the network through the outdoor terminal, and another laptop access the OLT as the monitor. Figure 6 shows the real-time video screenshot capture by the monitor, while the handheld terminal was on a car with the speed of $40 \mathrm{KM} / \mathrm{h}$. Figure 7 is the screenshots of three-way video call with two handsets and a laptop computer, which were 1.2KM away from the base station. 


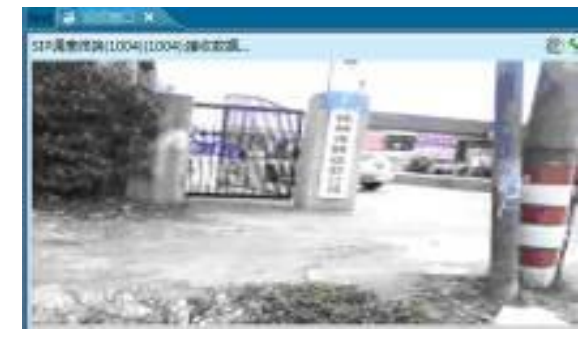

Figure 6. Real-time video screenshots with car move

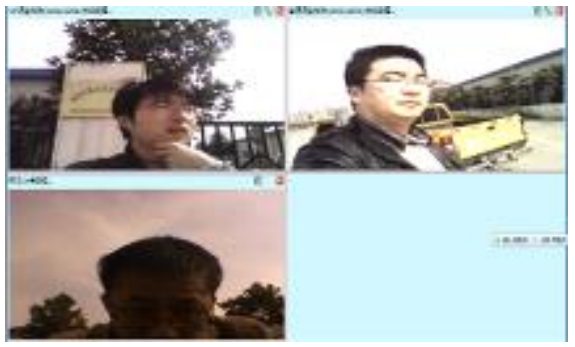

Figure 7. Three-way video calls screenshots

The test results show that, when the wireless base station transmission power is $1 \mathrm{~W}$, the power RoF system coverage radius is $1.2 \mathrm{KM}$ approximately.

With the same conditions, the fiber communication will need to excavate several roads to lay the fiber lines, the construction is very large. The EPON can be used as the backbone to connect the base stations to achieve multiple coverage areas, to settle the problems of traditional wireless communication, which needs the large network coverage and the security issues.

\section{SUMMARY}

RoF broadband access network is one of the best nextgeneration access network, also is one of the best choice for power communication access network. As there is no need fiber to each user, this structure can save the costs for FTTH network, and extend the effective access distance of the optical access (such as PON) and the wireless.

So the power RoF technology can provide broadband access services mainly include:

1) Feeder automation;

2) Automatic fault location;

3) Running multi-parameter monitoring on Distribution transformer;

4) High fault equipment monitoring and accident isolation

5) Data collection;

6) Integration communication of scheduling command: Dedicated fiber-optic and wireless network to establish an integrated command network scheduling communication platform, It could improve the emergency response capacity, network security and reliability. (Requires continuous coverage network)

7) Provide value-added services: such as mobile office system, etc. provide network services infrastructure. (Requires continuous coverage network)
Research on Traditional power communication access network provided a good starting for the design of hybrid RoF; however, setting up a RoF access system is more challenging than a single optical or wireless access network, because two completely different access technologies need to be considered together. Now electricity power RoF access technology needed to be in-depth study on the aspects of network establishment, connectivity and fault tolerance. Research on Traditional power communication access network provided a good starting for the design of hybrid RoF; however, setting up a RoF access system is more challenging than a single optical or wireless access network, because two completely different access technologies need to be considered together. Now electricity power RoF access technology needed to be in-depth study on the aspects of network establishment, connectivity and fault tolerance.

\section{ACKNOWLEDGMENT}

This article is completed by Nanjing IOT and IC design Innovation Center Ltd.. Great support in the software and hardware design, field experiments were given by colleagues.

Thanks the leaders and colleagues for the help.

\section{REFERENCES}

[1] $\mathrm{Xu}$ Kun, Li Jianqiang, the broadband wireless access of light load wireless system[M] Publishing house of electronics industry,2009

[2] Suman Sarkar, etc "Hybrid Wireless Optical Broadband Access Network(WOBAN) : A Review of Relevant Challenges" Journal of Light wave Technology, Vol.25. No.11. November 2007

[3] Zhang Hao, Pu Xiande, Guo Jinghong, Application of EPON Technology in Power Consumption Information Collection System, Telecommunications for electric power system,2010.vol31.7

[4] Xu Kun, Yin Jie, Li Jianqiang, Ultra-wideband RoF system and its key technology[J],ZTE Comunication,2009.3

[5] PIZZINAT A, LOURIKI I, CHARBONNIER B, et al. Low cost transparent radio-over-fiber system for UWB based home network [C] Proceedings of 34th European Conference on Optical Communication (ECOC'08), Sep 21-25, 2008, Brussels, Belgium. Piscataway, NJ, USA: IEEE, 2008.

[6] NGOMA A, SAUER M, GEORGE J, et al. Bit-rate doubling in multi-Gbps wideband ASK-modulated $60 \mathrm{GHz}$ RoF links using linear feed-forward equalisation and direct conversion transceivers[C]//Proceedings of 34th European Conference on Optical Communication (ECOC'08), Sep 21-25, 2008, Brussels, Belgium. Piscataway, NJ, USA: IEEE, 2008.

[7] Xie Chang, Zhang Baofu, Lai Xianzhu, Su Yang, Generating and Delivering Multi-Channel Local Oscillation Signals with High Purity in Radio-over-Fiber System Remotely[J],Chinese Journal Laser:2009.4

[8] Lu Jia, Chen Lin, Wen Shuangchun, Transmission Limitations and Corresponding Resolving Schemes in RoF Systems[J],ZTE Comunication:2009.3

[9] Bu Yang, Chen Youzhen,Primary Analysis of the Implement of Integration among the Telecom Cable and Internet on FTTx, Telecommunication network technology:2010.3

[10] TONG Ming-zhe, HU Zong-fu, GU Jie, LIU Tiring, Picocell seamless coverage based on adapting extended cell 